\title{
Tissue Doppler imaging: current and potential clinical applications
}

\author{
D J A Price, D R Wallbridge, M J Stewart
}

Echocardiography when combined with spectral and colour flow Doppler is well established as a safe, non-invasive, and versatile diagnostic modality in cardiology, and is now the predominant technique used for evaluation of left ventricular function and for the assessment and quantification of valvar heart lesions. When combined with physiological or pharmacological stress, echocardiography also enables the identification of reversible myocardial ischaemia and myocardial contractile reserve. ${ }^{12}$ However, assessment of regional cardiac dysfunction at rest and during stress remains subjective and semiquantitative, with high interobserver variability. ${ }^{3-6}$

Doppler measurement of myocardial motion, using pulsed wave Doppler, was first proposed in 1989 but this technique allowed real time visualisation of only a single myocardial segment and its potential was not realised. ${ }^{7}$ Only later, with the development of colour flow algorithms to visualise myocardial motion, did the technique begin to gain clinical acceptability. ${ }^{8-10}$ Subsequent software development has led to improved temporal and spatial resolution and off line processing to enable quantification of multiple segments of myocardium in seconds. These technological advances have been matched by widespread clinical interest in the technique and an explosion of clinical research. We review the current state of knowledge of tissue Doppler imaging, emphasising current clinical applications and likely future roles.

\section{Background}

Doppler tissue imaging uses the same principles as colour flow Doppler mapping, applying standard autocorrelation processing but reversing high velocity and low amplitude filters such that the high amplitude/low velocity motion of tissue is displayed in preference to blood flow. As cardiac structures move in a velocity range 0.06 to $0.24 \mathrm{~m} / \mathrm{s}$, some 10 times slower than myocardial blood flow, and have an amplitude approximately 40 decibels higher, it is possible to obtain images of tissue Doppler motion of high resolution without significant artefact originating from the blood pool. In such images, each pixel displays one colour representing a mean velocity value. However, comparison with pulsed wave Doppler traces of myocardial motion has indicated that initial Doppler tissue images failed to accurately quantify accurately each phase of the cardiac cycle. The normal cardiac cycle has four distinct peaks in systole and diastole (fig 1).
Cardiothoracic Division, South Cleveland Hospital, Middlesbrough, UK D J A Price M J Stewart

Royal Shrewsbury Hospitals NHS Trust, Shrewsbury, UK D R Wallbridge

Correspondence to: Dr M J Stewart, Consultant Cardiologist, Cardiothoracic Division, South Cleveland Hospital, Marton Road, Middlesbrough TS4 3BW, UK

michael.stewart@stahnhst. northy.nhs.uk

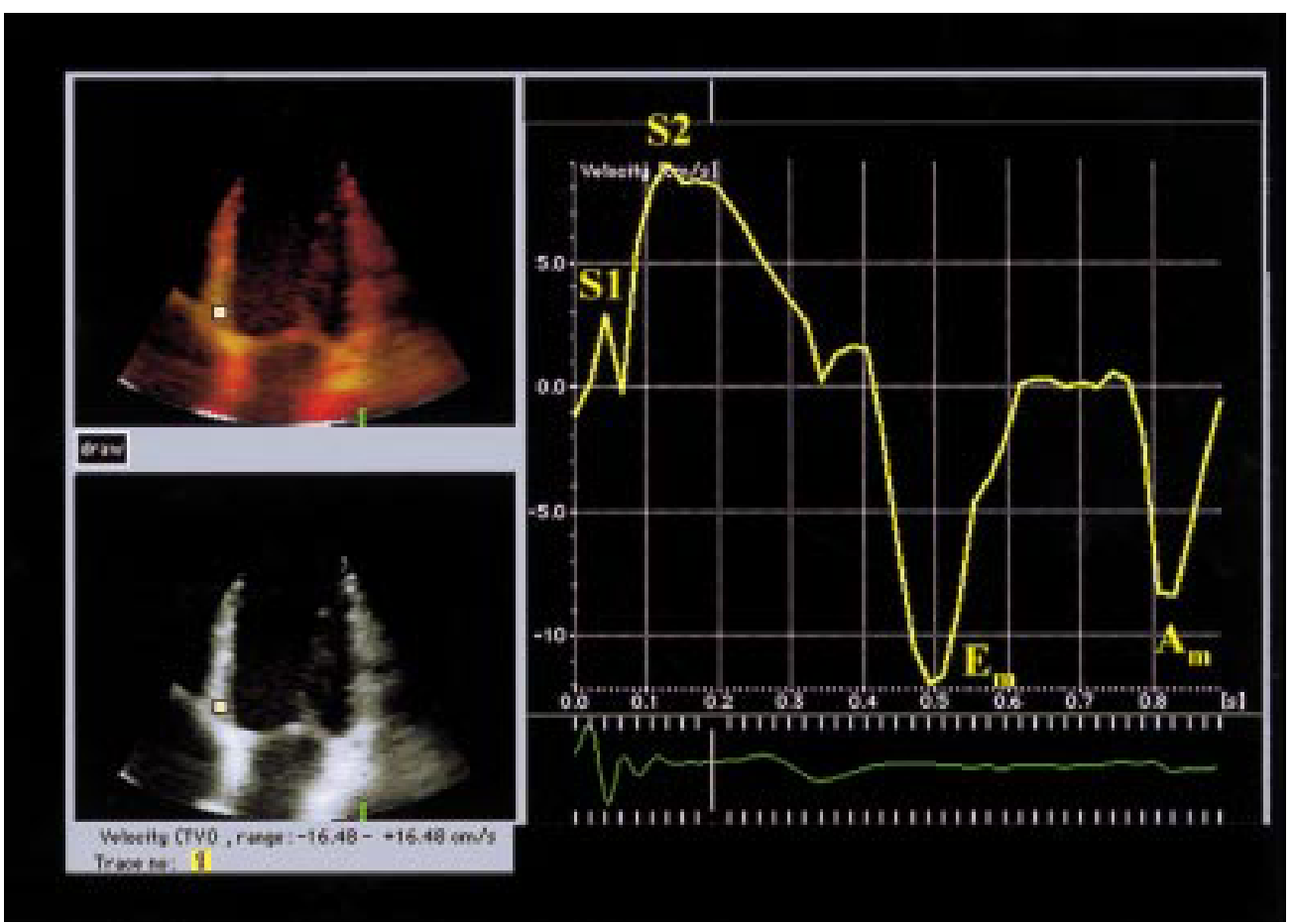

Figure 1 Normal myocardial velocity profile sampled from basal septum (apical four chamber view), showing typical pattern of two systolic and two diastolic peaks. S1, myocardial velocity associated with isovolumic contraction $(3.0 \mathrm{~cm} / \mathrm{s})$; $S 2$, peak systolic shortening velocity $(9.0 \mathrm{~cm} / \mathrm{s}) ; E_{m}$, indicates the peak early diastolic myocardial relaxation velocity $(-12.0$ $\mathrm{cm} / \mathrm{s}) ; A_{m}$, late diastolic myocardial velocity associated with atrial contraction $(-8.2 \mathrm{~cm} / \mathrm{s})$. 
The low frame rate $(25 \mathrm{~Hz})$ of initial Doppler tissue imaging systems provided a temporal resolution which was insufficient for accurate depiction of each phase of myocardial motion. Technological advances using digital parallel processing techniques now allow two dimensional (2D) Doppler tissue imaging with a $60^{\circ}$ sector of up to $80 \mathrm{~Hz}$. Combining such images with off line processing allows accurate quantification of regional myocardial motion in both systole and diastole, from multiple sites, with an image dataset being acquired within seconds.

\section{Doppler tissue imaging terminology}

In the original description of Doppler tissue imaging, three separate modalities were clearly distinguished. ${ }^{10}$ Myocardial Doppler velocity imaging uses standard colour coding to depict both velocity and direction of movement, with myocardial motion away from the transducer coded in blue, and towards the transducer coded in red. This bidirectional map allows semiquantification of myocardial motion. This can be displayed as a standard 2D image or as a Doppler tissue $M$ mode, where temporal resolution is greatly enhanced and differences in myocardial velocity from endocardium to epicardium can usually be appreciated. Recognition of this has led to the concept of the myocardial velocity gradient where the change in velocity from endocardium to epicardium is quantified. ${ }^{11}{ }^{12}$ By effectively quantifying the motion within a myocardial segment, this technique helps to eliminate errors arising from the inability to correct for translational motion of the heart within the chest and to reduce angle dependency. Recently, this concept has been developed further with $2 \mathrm{D}$ depiction of strain rate imaging.

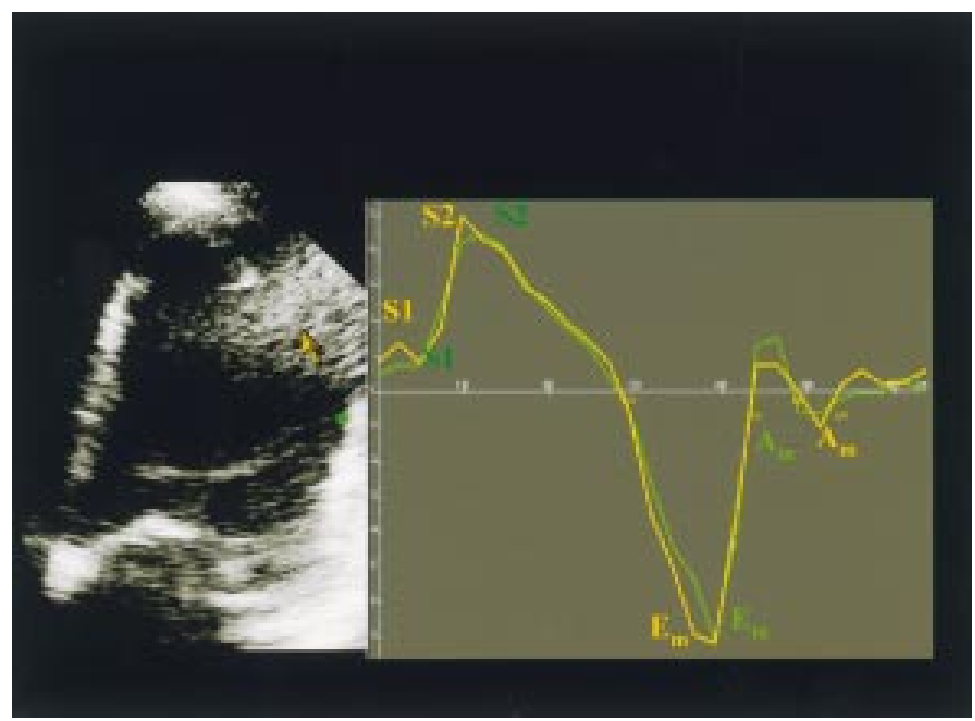

Figure 2 Part of a standard 2D apical four chamber view from a subject with suboptimal echo window, with myocardial velocity profiles shown from two discrete points in the lateral wall. The green trace shows the velocity profile from myocardium clearly defined on the $2 D$ image (green marker), and the yellow trace that from a nearby point where myocardium is not clearly discernible (yellow arrow). There is no significant difference between the myocardial velocity profiles, indicating that accurate tissue velocity data can still be obtained even in the absence of clear $2 D$ images. S1, myocardial velocity during isovolumic contraction; $S 2$, peak systolic myocardial velocity; $E_{m}$, peak early diastolic myocardial velocity; $A_{m}$, late diastolic myocardial velocity associated with atrial contraction.
Doppler acceleration mode imaging refers to a unidirectional map, where the change of velocity (or acceleration) from one frame to another is displayed in a $2 \mathrm{D}$ image according to a separate multicolour scheme. This technique has found some application in electrophysiology, enabling recognition of the site of pre-excitation in the Wolff-Parkinson-White syndrome. ${ }^{13-15}$

Doppler tissue energy mapping, now more frequently referred to as power Doppler imaging, has also been developed as part of this technique. The displayed tissue map represents the intensity of the power spectrum of the received Doppler signal, reflecting the composition of tissue. Hence, this map is both angle and velocity independent. This imaging modality has found a particular role in myocardial contrast echocardiography. Utilisation of autocorrelation processing means that, with the introduction of a left heart contrast agent which displays non-linear oscillation under ultrasound interrogation, there is increased variation in the Doppler energy signal, displayed as a notable increase in colour intensity. ${ }^{16}$ Use of Doppler energy mapping, combined with a left heart contrast agent, led to one of the first descriptions of contrast within the myocardium following intravenous injection in humans. ${ }^{17}$ With further technical development, combined with intermittent imaging and pulse inversion technology, this now forms the basis of much ongoing research in myocardial contrast technology. ${ }^{18} 19$

This review, however, concentrates on Doppler tissue velocity imaging and, to avoid confusion, this is referred to as tissue velocity imaging (TVI) hereafter.

\section{Development and normal values}

Initial validation of tissue velocity imaging, using rotating phantoms and a water bath, confirmed accurate representation of motion within the physiological range, but with over estimation and poor spatial resolution of velocity in very low range. ${ }^{10}$ Further phantom studies also documented the axial and lateral resolution of the $2 \mathrm{D}$ velocity map to be $3 \times 3 \mathrm{~mm}$, implying resolution sufficient for the documentation of a significant regional wall motion abnormality. While such resolution does not approach that obtained with standard 2D echocardiography, it is important to emphasise and appreciate that these images are entirely dependent on Doppler data, obtained from frequency shift information rather than from the reflected signal amplitude required for a grey scale image. As a result, tissue velocity data can be obtained even from subjects where the traditional echo window is suboptimal, and in particular means that wherever an image is obtained accurate quantification of myocardial motion is still reliable, in contrast to standard B-Mode imaging (fig 2).

As a Doppler based technique angle dependency remains a crucial issue, leading to the potential for error when trying accurately to quantify myocardial motion. As a result the technique as applied at present cannot reliably measure, for example, the velocity of contraction of the lateral wall in the parasternal short 
axis view. Contraction of mid-septum and posterior wall, which occurs parallel to the scan line, can be accurately assessed. However, at the much lower velocities encountered with tissue motion, the contribution of overall cardiac translational motion within the thorax becomes important resulting, for example, in underestimation of septal systolic velocity and overestimation of posterior wall systolic velocity in parasternal views, because of movement of the heart towards the transducer. These limitations may explain, in part, the relatively poor reproducibility of this aspect of the technique. ${ }^{20}$ While appearing a significant limitation of tissue velocity imaging, this effect may be minimised when measurement of velocity is relative to other myocardial regions, or of different points within the same region, rather than relative to the ultrasound transducer. This theory led to the development of the transmyocardial velocity gradient, for which normal values have been proposed, ${ }^{11}{ }^{12} 21$ and more recently to myocardial strain rate imaging. ${ }^{22}$ Although still under evaluation, these developments should facilitate the application of TVI technology to imaging patients with ischaemic heart disease.

From the cardiac apex, the impact of translational motion of the heart within the thorax becomes much less important. As myocardial thickening is occurring predominantly at right angles to the direction of Doppler interrogation from the apex, this cannot reliably be measured with tissue velocity imaging. However, longitudinal shortening velocities do appear to be reliably measured from the apical window. Normal myocardial motion is complex, with three separate components: radial contraction, longitudinal shortening, and rotation. At present, no echo technique can simultaneously assess all three components and there is debate about the relative importance of each. There is now little doubt, however, that longitudinal shortening is an integral part of global contractile function, and it is thought to play as important a role as radial thickening in contributing to left ventricular (LV) ejection fraction. ${ }^{23-26}$ The potential for quantification of longitudinal shortening as a simple estimate of overall systolic function has recently been proposed. ${ }^{27-31}$ Tissue velocity imaging from the apex has the potential to provide equivalent and perhaps more extensive clinical information more readily and more reproducibly. ${ }^{32} 33$

Longitudinal contraction and relaxation velocities of the LV myocardium are greatest in the basal segments and decrease progressively toward the apex, where in fact velocities may become reversed. ${ }^{34}$ This is consistent with previous observations that $\mathrm{LV}$ contraction occurs towards a central point situated two thirds along a long axis line from the level of the mitral annulus to the apex. ${ }^{35}$ The normal longitudinal myocardial velocity profile when imaged from the apex consists of a systolic phase directed toward the transducer, and a diastolic phase directed away. The systolic phase consists of an early peak coinciding with isovolumic contraction followed by a second, larger peak coinciding with descent of the cardiac base. The normal diastolic phase consists of two distinct peaks, the first early $\left(\mathrm{E}_{\mathrm{m}}\right)$ and the second late $\left(\mathrm{A}_{\mathrm{m}}\right)$ in diastole. These correspond respectively to early and late $\mathrm{LV}$ diastolic relaxation, and thus to early and late ventricular filling, resembling in relative magnitude the $\mathrm{E}$ and $\mathrm{A}$ waves of the mitral inflow velocity profile measured by pulsed wave Doppler (fig 1).

Normal values for longitudinal shortening have been reported as mean (SD) 7.5 (1.3) to $9.5(1.4) \mathrm{cm} / \mathrm{s}$ for the anterior septum, and $10.3(1.9) \mathrm{cm} / \mathrm{s}$ for the basal lateral wall. ${ }^{36} 37$ The highest systolic tissue velocities in the long axis are observed in the mitral annulus and the basal segments of the lateral, anterior, and posterior free walls, followed by those in the basal septum. Peak early diastolic velocities also vary between segments, with highest values in the posterior and lateral walls $(16.5$ (4.0) to 17.5 $(2.9) \mathrm{cm} / \mathrm{s})$ and lowest in the anterior septum (10.9 (2.4) to $12.9(3.1) \mathrm{cm} / \mathrm{s})$. With the exception of the anterior and posterior septum, diastolic lengthening velocities are significantly higher in the basal segments than the mid wall segments. The ratio of early diastolic to late diastolic velocities $\left(\mathrm{E}_{\mathrm{m}} / \mathrm{A}_{\mathrm{m}}\right)$ in normal subjects is similar to the conventional $\mathrm{E} / \mathrm{A}$ ratio of mitral inflow velocities. ${ }^{38}$ Studies aiming to determine normal values and including both male and female subjects have been limited by relatively small numbers. Although there is little evidence thus far of a significant difference in velocities between the sexes, increasing age has been associated with a gradual fall in myocardial velocities both in systole and diastole. ${ }^{37}{ }^{39}$

TVI has also demonstrated differences within myocardial segments. $M$ mode studies, obtained from the parasternal window, in a limited number of segments, have shown an intramyocardial velocity gradient with a graduated increase in radial shortening velocity from the subepicardial to the subendocardial region. ${ }^{11}{ }^{12}$ When imaging from the apex, there is also a quantifiable velocity gradient between two points separated in the longitudinal axis but lying within the same segment, a feature which may lend itself to assessment of regional contractile function. ${ }^{22}$ Recognition of these intramyocardial velocity gradients has led to the suggestion that persistence could represent viability in an otherwise akinetic segment; a hypothesis which requires further confirmatory work.

\section{Systolic function}

One of the first clinical applications of TVI has been as a method of assessing left ventricular systolic function. Assessment of this parameter remains the commonest indication for echocardiography, but standard methods are often limited by technical difficulties, inaccuracy and poor reproducibility. ${ }^{2940}{ }^{41}$ The advantages of a Doppler based technique as a means of acquiring data make this an attractive alternative to standard 2D imaging, and there is already evidence that TVI applications offer new and easy methods of assessing both global and regional LV systolic function. 
GLOBAL LV FUNCTION

Descent of the cardiac base towards the ventricular apex is a feature of normal ventricular systolic function. This has been quantified by measuring the displacement distance of the mitral annulus by $M$ mode echocardiography and correlates with left ventricular ejection fraction. ${ }^{25}{ }^{27}$ It follows that the rate of mitral annular displacement may also reflect LV systolic function and studies using TVI to measure mitral annular displacement velocity (MADV) confirm this. ${ }^{32}$ Measurement is made at several sites - for example, lateral, septal, inferior, and anterior aspects of the annulus - to derive an average figure for MADV, as an adjacent regional wall motion abnormality may affect localised values and a mean velocity is more likely to reflect global LV function. Measurement of MADV is relatively easy as, even in difficult echo subjects, tissue velocity images of the mitral annular region can almost always be obtained from the apical window. On the 2D tissue Doppler map, the sample volume for PWTDS is placed over the mitral annulus and TVI software then allows real time readout of the velocity changes of that region with time. Alternatively, a cineloop can be stored digitally for offline analysis, where computer software allows reconstruction of velocity profiles in graphical form from any point on the tissue Doppler map.

REGIONAL LV FUNCTION

Positioning a sample volume within the myocardium equidistant from endocardial and epicardial borders allows measurement of segmental myocardial velocities, both at rest and with pharmacological stress. Regional velocities can be measured both in real time using PWTDS, or can be analysed offline by storing a digital cineloop. Modern systems capable of high frame rates, and thus high temporal resolution, ensure that velocity profiles derived from a stored cineloop closely reflect those obtained by PWTDS. This allows rapid acquisition of sufficient data for analysis of multiple regions simply by acquiring one cineloop, obviating the need for acquisition of multiple regional samples by PWTDS.

The colour coded 2D tissue velocity map also lends itself to the assessment of regional function, as deviations from the normal pattern of LV motion and colour changes throughout the cardiac cycle are often readily appreciated, providing immediate online appreciation of cardiac asynchrony. Areas of akinesia after myocardial infarction, for example, are shown by darker hues of colour representing low velocity of motion (fig 3), and dyskinetic segments may be seen to exhibit colours opposite to those of adjacent normal segments, particularly during systole.

Analysis of the intramyocardial velocity gradients also aids detection of regional dysfunction. M mode studies reveal reduction or loss of the subendocardial-subepicardial gradient during ischaemia or infarction, though the restriction of this technique to only a few myocardial segments reduces its utility as a practical tool. ${ }^{42}$ In contrast, the ability to measure

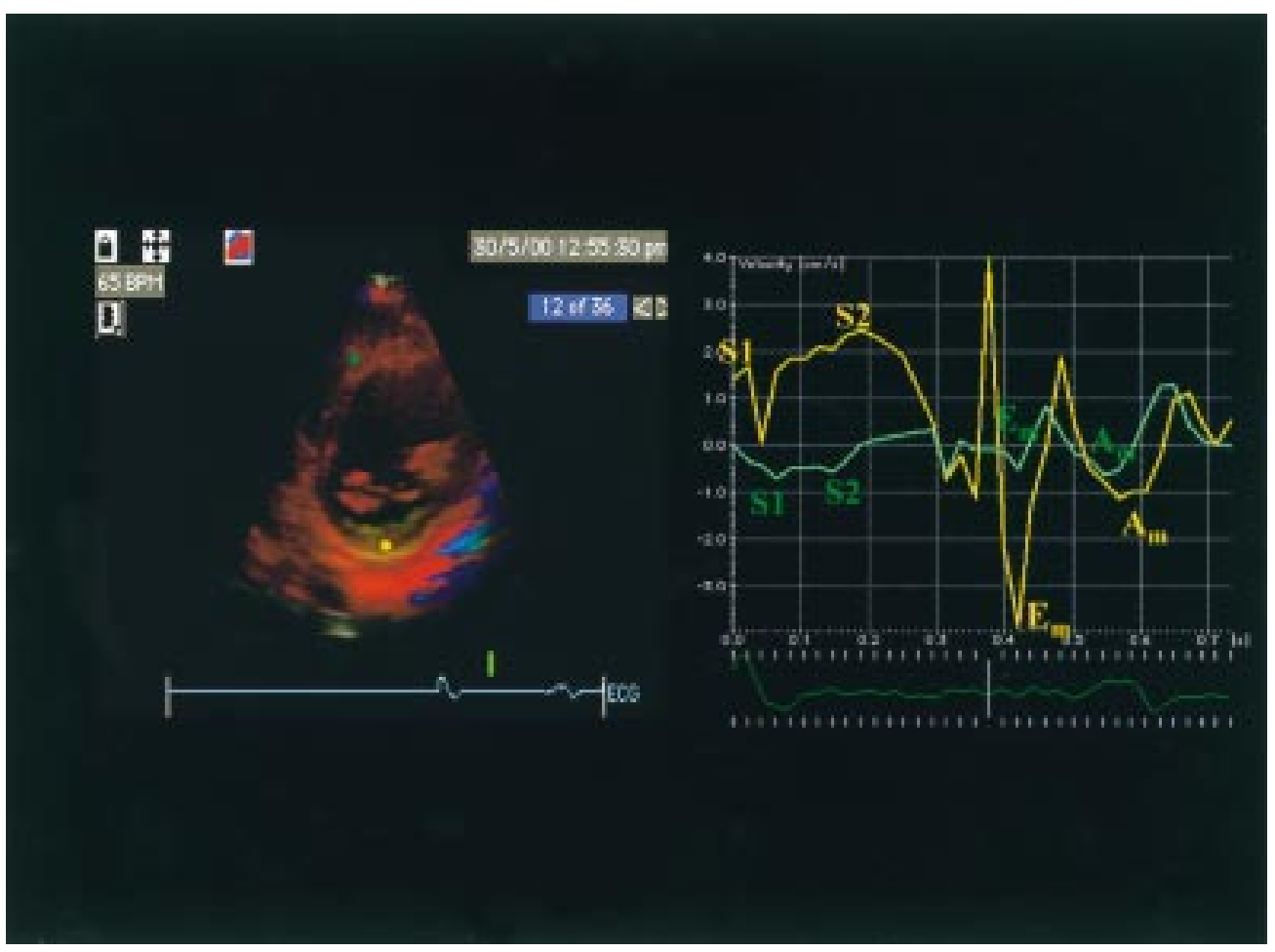

Figure 3 Tissue velocity map superimposed on a 2D parasternal short axis view from a patient with recent antero-septal myocardial infarction. Myocardial velocity profiles are shown from the mid-septum (green marker and line) and mid-posterior wall (yellow marker and line). The peak systolic contraction velocity of the posterior wall is within the normal range (yellow $S 2=2.4 \mathrm{~cm} / \mathrm{s}$ ) whereas the corresponding velocity from the septum is minimal ( $\mathrm{green} S 2=-0.6 \mathrm{~cm} / \mathrm{s}$ ), indicating akinesis. Diastolic velocities in the akinetic septal segment are similarly reduced. $S 1$, myocardial velocity during isovolumic contraction; $S 2$, peak systolic myocardial velocity; $E_{m}$, peak early diastolic myocardial velocity; $A_{m}$, late diastolic myocardial velocity associated with atrial contraction. 


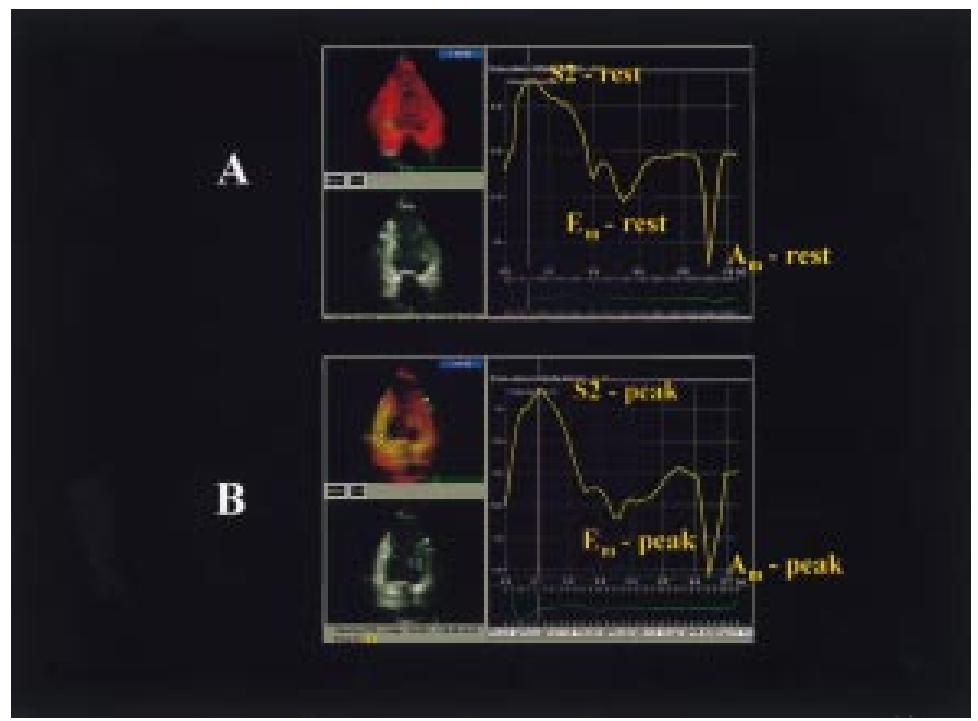

Figure 4 Myocardial velocity profiles obtained from the basal inferior segment (apical 2 chamber view) recorded at rest (panel $A$ ) and after full dose dobutamine stress (panel B). The systolic profile shows fusion of $S 1$ and $S 2$ (which often occurs, especially with rapid heart rates) and demonstrates an increase in peak systolic velocity, $S 2$, from $8.0 \mathrm{~cm} / \mathrm{s}$ to $13.0 \mathrm{~cm} / \mathrm{s}$ in a subject with normal response. In this example the diastolic velocities $E_{m}$ and $A_{m}$ are also seen to increase with dobutamine stress (from -5.5 to -7.1 and from -12.8 to $-15.8 \mathrm{~cm} / \mathrm{s}$ respectively), the significance of which remains to be established. S1, myocardial velocity during isovolumic contraction; $S 2$, peak systolic myocardial velocity; $E_{m}$, peak early diastolic myocardial velocity; $A_{m}$, late diastolic myocardial velocity associated with atrial contraction. angle correction. Thus, velocities measured relative to the transducer must be interpreted with caution when trying to assess the systolic function of individual segments.

\section{Stress echocardiography}

Normal subjects show an increase in peak systolic velocity with exercise or dobutamine stress (fig 4), with an attenuated response in patients with impaired coronary flow reserve. ${ }^{44-48}$ TVI provides an alternative to conventional semiquantitative methods for the diagnosis and assessment of ischaemic heart disease by stress echocardiography. Although limited by many of the drawbacks which apply to its use at rest (angle dependency, heart translational motion, and the influence of adjacent myocardial segments on velocities measured in the segment of interest) it does appear to introduce greater objectivity in regional quantification and thus improve reproducibility, overcoming some of the limitations of conventional stress echocardiography. ${ }^{49} 50$

Initial work involved use of PWTDS, requiring real time data acquisition from multiple segments during a stress echo study, thus limiting its practical utility. ${ }^{46}$ However, the development of new ultrasound systems with the ability to acquire real time colour Doppler images for subsequent offline analysis, with sufficiently high temporal resolution to ensure accurate measurement of peak velocity, has made the application of TVI methods to stress echocardiography a practical reality. ${ }^{44} 51$

Early attention focused on measurement of peak systolic velocity as the parameter likely to be most useful in assessing regional LV systolic function with stress, but more recently other parameters such as the time to peak systolic velocity and the velocity time integral have been proposed as equally important measures. ${ }^{49}$ The multicentre, multinational MYDISE study (MYocardial Doppler In Stress Echocardiography) is an ongoing study assessing the feasibility and reproducibility of these TVI methods. Early results indicate that TVI does enhance both objectivity and reproducibility of stress echocardiography. ${ }^{50}$

Furthermore, the susceptibility of subendocardial longitudinal muscle fibres to early ischaemia suggests that longitudinal shortening velocity measured from the apical window should be a sensitive marker of impaired contractile function-perhaps more so than recognition of reduced endocardial excursion, on which conventional assessment by stress echo traditionally depends. ${ }^{52}$ Longitudinal shortening velocities measured from the apex are reported to show greater response to dobutamine and to be more reproducible than radial contraction velocities measured from the parasternal window. ${ }^{49}$ This enhancement of stress echocardiographic methods by use of TVI applications may be improved still further if developments in strain rate imaging allow incorporation of this technique in future. plane can be measured without the need for 


\section{Diastolic function}

TVI holds great promise for the assessment of LV diastolic function. Whereas conventional Doppler measures of LV filling derived from mitral inflow velocities reflect only global diastolic function, TVI offers the ability to measure regional diastolic function by echocardiography for the first time. Spectral PWTDS allows quantification of diastolic myocardial velocities from a small sample volume placed in a region of interest in the same way as for systolic velocities, and this has enabled characterisation of the normal pattern of early and late diastolic velocity peaks $\left(\mathrm{E}_{\mathrm{m}}\right.$ and $A_{m}$ respectively), with highest values at the mitral annulus, decreasing progressively towards the apex. ${ }^{36}$

Relaxation velocity of the mitral annulus, averaged from four different sites, reflects global LV diastolic function and correlates (in normal subjects) with conventional measures derived from LV filling patterns and the time constant of isovolumic relaxation $(\tau) .^{53-55} \mathrm{Re}-$ gional myocardial diastolic velocities, measured as segmental longitudinal relaxation velocities from the apical window, are relatively uninfluenced by translational motion although-as for regional systolic functioncare must be taken when interpreting localised values in view of the possible influence of adjacent segments. A conventional measure of global diastolic function, E/A ratio, has been shown to fall with an increase in the number of myocardial segments exhibiting a reduction in the tissue velocity $\mathrm{E}_{\mathrm{m}} / \mathrm{A}_{\mathrm{m}}$ ratio, lending support to the hypothesis that TVI offers a useful measure of regional diastolic function. ${ }^{38}$ Moreover, there is evidence that diastolic tissue velocities are less influenced by the changes in preload which commonly compensate for diastolic dysfunction and which confound assessment by standard measures based on LV filling patterns. ${ }^{54-56}$

With normal LV filling, the peak early diastolic myocardial velocity $\left(\mathrm{E}_{\mathrm{m}}\right)$ measured in the long axis plane is $>10 \mathrm{~cm} / \mathrm{s}$ in the young, falling to $>8 \mathrm{~cm} / \mathrm{s}$ with aging. Both early and intermediate stages of diastolic dysfunction (the delayed relaxation and pseudonormal phases respectively), are characterised by a reduction in $\mathrm{E}_{\mathrm{m}}$ velocity to $<8 \mathrm{~cm} / \mathrm{s}$, falling further still in the most severe, or restrictive, stage.$^{54}$ Hence myocardial velocities are persistently reduced even in those stages of diastolic dysfunction characterised by increased preload compensation and thus normal or high mitral inflow velocities. This lack of concordance between the two parameters in the pseudonormal and restrictive stages means that myocardial velocity measurement can allow better recognition of these patterns, which are otherwise often difficult to distinguish from normal.

This has practical value in differentiating restrictive cardiomyopathy from pure constrictive physiology, a difficult distinction when relying on standard $2 \mathrm{D}$ and Doppler criteria alone. Garcia et al have reported that a tissue Doppler $\mathrm{E}_{\mathrm{m}}$ velocity of $8 \mathrm{~cm} / \mathrm{s}$ reliably separates restrictive cardiomyopathy (characterised by a low $\mathrm{E}_{\mathrm{m}}$ value in the context of "pseudonormal- ised" LV filling with high E velocity and a normal E/A ratio) from constrictive physiology (normal/high $\mathrm{E}_{\mathrm{m}}$ velocity if systolic function remains normal). ${ }^{57}$

Mitral inflow velocity patterns also differ from diastolic myocardial velocity patterns in some groups with ventricular hypertrophy, enabling differentiation between the physiological hypertrophy of athletes and pathological hypertrophy of hypertrophic cardiomyopathy (HCM), for example. Palka et al reported that myocardial velocity gradient (MVG), measured across the posterior wall of the LV in the parasternal short axis view, discriminated between patients with HCM and those with hypertensive LV hypertrophy $(\mathrm{LVH})$ or athletic hearts. ${ }^{58}$ There was no significant difference in the conventional Doppler indices of LV filling to differentiate the HCM and the athletic LVH groups, suggesting that myocardial $\mathrm{E}_{\mathrm{m}}$ velocities are less influenced by alterations in preload, which can confound assessment of diastolic function by conventional LV filling indices. Further evidence of the relative preload independence of $\mathrm{E}_{\mathrm{m}}$ velocity was presented by Oki et al, who reported that the time constant of isovolumic LV relaxation $(\tau)$ correlated well with $E_{m}$ velocities regardless of $\mathrm{LV}$ filling pressure. ${ }^{55}$ Sohn et al also demonstrated that manipulation of LV filling dynamics produced alterations in the LV Doppler inflow patterns but with no significant changes in the $E_{m}$ velocities. ${ }^{56} \mathrm{E}_{\mathrm{m}}$ velocity thus has a unimodal distribution, Doppler myocardial velocities showing a steady decline with increasing diastolic dysfunction, in contrast to the bimodal distribution of mitral inflow velocity $E$ which occurs due to preload compensation in the more severe stages. Again, these observations are consistent with relative independence of $\mathrm{E}_{\mathrm{m}}$ in relation to compensatory alterations in loading conditions..$^{534}$

Assessment of diastolic function by conventional Doppler echocardiography relies heavily on study of the mitral inflow velocity profile, but this is of limited value in the presence of atrial fibrillation or other arrhythmia. As TVI enables the study of diastolic function by direct measurement of myocardial motion, it does not suffer this limitation of conventional methodology. ${ }^{59}$

Reversible myocardial ischaemia affects diastolic function earlier than systolic function suggesting that regional diastolic function measurement could aid its assessment. ${ }^{60}$ PWTDS has enabled rapid and accurate measurement of both myocardial diastolic velocities and regional isovolumic relaxation times, which have been used to differentiate between normally perfused and ischaemic segments. ${ }^{78}{ }^{31}$ Myocardial ischaemia is characterised by a reduction in $\mathrm{E}_{\mathrm{m}}$ velocity and consequently also in $\mathrm{E}_{\mathrm{m}} / \mathrm{A}_{\mathrm{m}}$ ratio. ${ }^{3845}$ The $\mathrm{A}_{\mathrm{m}}$ velocity however has been reported to remain unchanged, which may be because whereas early diastolic changes reflect an active, energy requiring process, late diastolic filling merely reflects passive stretch after atrial contraction. ${ }^{3861}$ Full clinical utility of these 


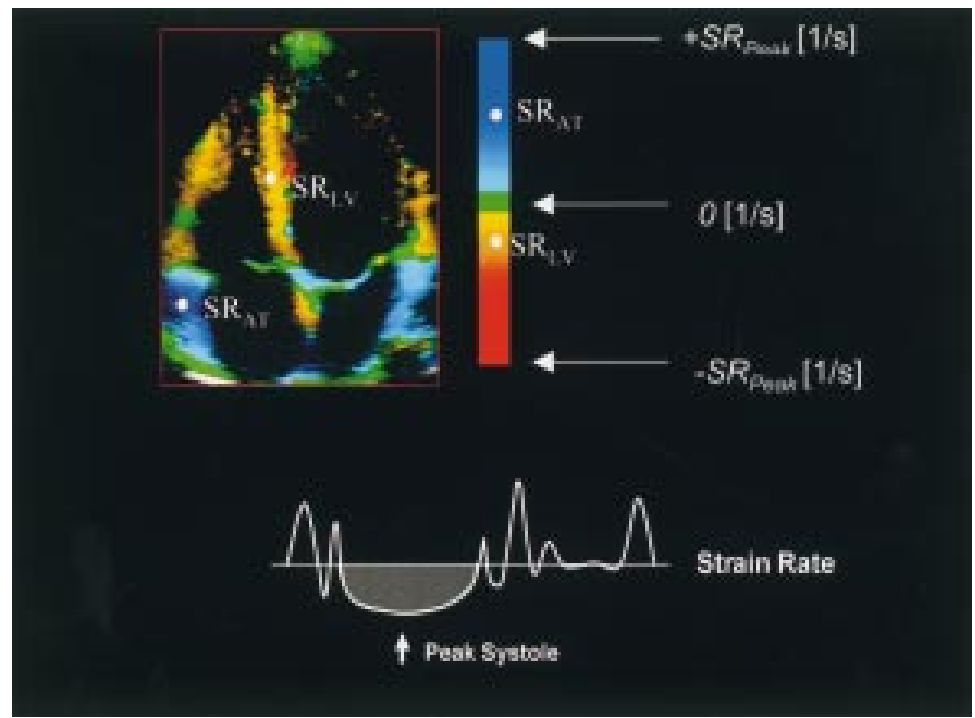

Figure 5 Colour map superimposed upon an apical four chamber view to represent myocardial strain rate. Myocardial contraction is associated with a negative strain rate and is coloured orange-red, whereas myocardial expansion is coloured blue. In this frame, captured during ventricular systole, the contracting ventricular myocardium is coloured predominantly orange, as for example at point $S R_{L V}$, and the relaxing atrial myocardium is coloured blue, as at point $S R_{A T}$. Strain rate is expressed as units/second, but the normal range is yet to be established. The colour bar therefore depicts the range of strain rates, where $+S R_{P_{\text {eal }}}$ represents a maximally positive strain rate (maximum myocardial expansion), and $S R_{\text {Peak }}$ represents a maximally negative strain rate (maximum myocardial contraction), without absolute values.
The lower part of the panel shows a graphical representation of changes in strain rate during the cardiac cycle; this can be seen to be approximately a mirror image of the changes in myocardial velocity. The hatched area during ventricular systole represents the integral of myocardial strain rate which gives the myocardial strain.

\section{Summary}

Tissue velocity imaging is another important development in the field of cardiac ultrasound and promises a number of applications to further extend the unique diagnostic role of echocardiography. Accurate quantification of global and particularly regional systolic LV function is closer than ever before, and the promise to improve the reproducibility and diagnostic value of stress echocardiography looks set to be realised, though full results from MYDISE are eagerly awaited.

Tissue velocity imaging has enabled echocardiographic assessment of regional diastolic function which appears to be less limited by the compensatory changes in loading conditions that confound measurement of diastolic function by conventional echocardiographic methods. Tissue velocity imaging thus offers a practical clinical tool to differentiate physiological from pathological LV hypertrophy, and restrictive from constrictive physiology by refreshingly simple means.

The development of the new datasets strain and strain rate promise to further enhance the value of echocardiography by overcoming some of the limitations of early TVI techniques; this should allow not only superior assessment of regional contractile function at rest and with stress, but also reliable identification of viable myocardium. quantification of regional myocardial ischaemia, overcoming some of the limitations of conventional TVI. Doppler measurement of myocardial strain has resulted from the further development of TVI principles and allows quantification of myocardial shortening within an individual segment (fig 5). This represents a more logical method of assessing regional contractile function as these datasets are not influenced by the function of adjacent myocardial segments and are less dependent upon the direction of shortening in relation to the transducer. Strain rate is a measure of the velocity of deformation of myocardium, and is given by the formula $(V 2-V 1) / d$, where $V 1$ and $V 2$ are velocities of myocardial shortening at two points separated by distance $d$; this gives the difference in tissue velocity per unit length. ${ }^{22}$ Integration of this parameter with respect to time gives myocardial strain, a measure of the per cent compression of myocardium during systole. $^{22}$ This is also given by the formula $(L 1-L 2) / L 1$, where $L 1$ is the distance between two points at end diastole and $L 2$ is the distance between the same two points at end systole; this parameter cannot otherwise be measured directly by $2 \mathrm{D}$ echo without placement of myocardial markers. This approach will clearly allow identification of akinetic segments, where strain rate and strain will be zero, and may also allow identification of inducible ischaemia and viable myocardium. ${ }^{62-65}$
The authors are grateful to $\mathrm{Mr}$ Simon Robson of IGE Medical Systems Limited for help with fig 5. Dr DJA Price is supported by a grant from the South Cleveland Heart Fund.

1 Geleijnse ML, Fioretti PM, Roelandt JRTC. Methodology, stress echocardiography. $\mathcal{F}$ Am Coll Cardiol 1997;30:595606.

2 Perrone-Dilardi P, Pace L, Prastaro M, et al. Dobutamine echocardiography predicts improvement of hypoperfused dysfunctional myocardium after revascularisation in pa2556-65.

3 Hoffman R, Lethen H, Marwick T, et al. Analysis of interinstitutional observer agreement in interpretation of $1996 ; 27: 330-6$

4 Hoffman R, Hanrath P. Stress echocardiography: the scourge of subjective interpretation. Eur Heart $\mathcal{F}$ 1995;16: 1458-9.

5 Picano E, Lattanzi F, Orlandini A, et al. Stress echocardiography and the human factor: the importance of being an expert. F Am Coll Cardiol 1990;17:666-7.

6 Iliceto S, Galiuto L, Marangelli V, et al. Clinical use of stress echocardiography: factors affecting diagnostic accuracy. Eur Heart f 1994;15:672-80.

7 Isaaz K, Thompson A, Ethevenot G, et al. Doppler echocardiographic measurement of low velocity motion of the left ventricular posterior wall. Am F Cardiol 1989;64:66-75.

8 McDicken WN, Sutherland GR, Moran CM, et al. Colour doppler velocity imaging of the myocardium. Ultrasound Med Biol 1992;18:651-4.

9 Yamazaki N, Mine Y, Sano A, et al. Analysis of ventricular wall motion using color-coded tissue Doppler imaging system. Fpn F Appl Phys 1994;33:3141-6. et al. Colour Doppler myocardial imaging: a new technique et al. Colour Doppler myocardial imaging: a new technique
for the assessment of myocardial function. F $\mathrm{Am}$ Soc
Echocardiogr 1994; 7:441-58.

11 Fleming AD, Xia X, McDicken WN, et al. Myocardial velocity gradients detected by doppler imaging. Br $\mathrm{F}$ Radiol 1994;67:679-88. feasibility, safety and diagnostic accuracy of dobutamine tients with coronary artery disease. Circulation 1995;92: dobutamine stress echocardiogams. I Am Coll Cardiol

10 Sutherland GR, Stewart MJ, Groundstroem KWE, 
12 Uematsu M, Miyatake K, Tanaka N, et al. Myocardial velocity gradient as a new indicator of regional left

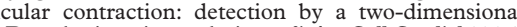
tissue Doppler imaging technique. F Am Coll Cardiol 1995 26:217-23

13 Nakayama K, Miyatake K, Uematsu M, et al. Application of tissue Doppler imaging technique in evaluating early ventricular contraction associated with accessory atrioventricular pathways in Wolff-Parkinson-White syndrome. $A m$ Heart f 1998;135:99-106.

14 Tuchnitz A, Schmitt C, Von Bibra H, et al. Non-invasive localisation of accessory pathways in patients with
Wolff-Parkinson-White syndrome with the use of myocardial Doppler imaging. F Am Soc Echocardiogr 1999;12 $32-40$.

15 Fedele F, Trambaiolo P, Magni G, et al. New modalities of regional and global left ventricular function analysis: state of the art. Am $₹$ Cardiol 1998;81 (suppl 12A):49G$57 \mathrm{G}$.

16 Hudson-Dixon CM, Long BW, Cox LA. Power Doppler imaging: principles and applications. Radiologic Technology 1999;70:235-43.

17 Stewart MJ, Sutherland GR, Groundstroem KWE, et al. Colour Doppler imaging of the myocardium following intravenous injection of a transpulmonary echo contras agent-a new technique to evaluate regional myocardia perfusion [abstract]. Eur Heart $\mathcal{F}$ 1993;14:1124

18 Tuchnitz A, Von Bibra H, Sutherland GR, et al. Doppler energy: a new acquisition technique for the transthoracic detection of myocardial perfusion defects with the use of a venous contrast agent. F Am Soc Echocardiogr 1997;10:88190.

19 Tiemann K, Lohmeier S, Kuntz S, et al. Real-time contrast echo assessment of myocardial perfusion at low emission power: first experimental and clinical results using power pulse inversion imaging. Echocardiography 1999;16:799810.

20 Vinereanu D, Khokhar A, Fraser AG. Reproducibility of pulsed wave tissue Doppler echocardiography. $\mathcal{F} \mathrm{Am} S o c$ pulsed wave tissue Doppler
Echocardiogr 1999;12:492-9.

21 Palka P, Lange A, Fleming AD, et al. Age-related transmural peak mean velocities and peak velocity gradients by Doppler myocardial imaging in normal subjects. Eur Hear f 1996; 17:940-50

22 Heimdal A, Stoylen A, Torp H, et al. Real-time strain rate imaging of the left ventricle by ultrasound. I $\mathrm{Am}$ So Echocardiogr 1998;11:1013-19.

23 Greenbaum RA, Ho SY, Gibson DG, et al. Left ventricula fibre architecture in man. Br Heart f 1981;45:248-63.

24 Jones CJ, Raposo L, Gibson DG. Functional importance of the long axis dynam

25 Alam M, Rosenhamer G. Atrioventricular plane displacement and left ventricular function. $\mathcal{F} \mathrm{Am}$ Soc Echocardiogr 1992;5:427-33.

26 Lundbeck S. Cardiac pumping and function of the ventricular septum. Acta Physiol Scand Suppl 1986;550:1101

27 Alam M, Hoglund C, Thorstrand C. Longitudinal systolic shortening of the left ventricle: an echocardiographic study in subjects with and without preserved global function. Clin Physiol 1992;12:443-52.

28 Alam M. The atrioventricular plane displacement as a means of evaluating left ventricular systolic function in acute myocardial infarction. Clin Cardiol 1991;14:588-94.

29 Simpson IA. Echocardiographic assessment of long axis function: a simple solution to a complex problem? Heart 1997;78:211-12

30 Simonson JS, Schiller NB. Descent of the base of the left ventricle: an echocardiographic index of left ventricular function. F Am Soc Echocardiogr 1989;2:25-35.

31 Alam M, Hoglund C, Thorstrand C, et al. Haemodynamic significance of the atrioventricular plane displacement in patients with coronary artery disease. Eur Heart f 1992;13: 194-200.

32 Gulati V, Katz WE, Follansbee WP, et al. Mitral annular descent velocity by tissue doppler echocardiography as an descent velocity by tissue doppler echocardiography as an
index of global left ventricular function. Am $\mathfrak{f}$ Cardiol index of global

33 Sutherland GR, Lange A, Palka P, et al. Does doppler myocardial imaging give new insights or simply old information revisited? Heart 1996; 76:197-9.

34 Erbel R, Wallbridge DR, Zamorano J, et al. Tissue doppler echocardiography. Heart 1999;76:193-6.

35 Ingels NB, Daughters II GT, Stinson EB, et al. Evaluation of methods for quantitating left ventricular segmental wall motion in man using myocardial markers as a standard. Circulation 1980;61:966-72.

36 Galiuto L, Ignone G, DeMaria AN. Contraction and relaxation velocities of the normal left ventricle using pulsedwave tissue doppler echocardiography. Am 7 Cardiol 1998; 81:609-14.

37 Alam M, Wardell J, Andersson E, et al. Characteristics of mitral and tricuspid annular velocities determined by pulsed wave Doppler tissue imaging in healthy subjects. $\mathscr{f}$ Am Soc Echocardiogr 1999;12:618-28.

38 Garcia-Fernandez MA, Azevedo J, Moreno $\mathrm{M}$, et al. Regional diastolic function in ischaemic heart disease using pulsed wave doppler tissue imaging. Eur Heart $\mathcal{F}$ 1999;20 496-505.
39 Onose Y, Oki T, Mishuno Y, et al. Influence of aging on systolic left ventricular wall motion velocities along the long and short axes in clinically normal patients determined by pulsed tissue Doppler imaging. F Am Soc Echocardiogr 999;12:921-6.

40 Schiller NB, Foster E. Analysis of left ventricular systolic function. Heart 1996;75(suppl 2):17-26.

41 Schiller N, Shah PM, Crawford M, et al. Recommendations for quantitation of the left ventricle by two-dimensional echocardiography. F Am Soc Echocardiogr 1989;2:358-67.

42 Tsutsui H, Uematsu M, Shimizu H, et al. Comparative usefulness of myocardial velocity gradient in detecting ischemic myocardium by a dobutamine challenge. $7 \mathrm{Am}$ Coll Cardiol 1998;31:89-93.

43 Miyatake K, Yamagishi M, Tanaka N, et al. New method for evaluating left ventricular wall motion by color-coded tissue Doppler imaging: in vitro and in vivo studies. $\mathcal{F} \mathrm{Am}$ Coll Cardiol 1995;25:717-24.

44 Wilkenshoff UM, Sovany A, Wigstrom L, et al. Regional mean systolic myocardial velocity estimation by real-time colour Doppler myocardial imaging: a new technique for quantifying regional systolic function. $\mathcal{F} \mathrm{Am} \mathrm{Soc}$ Echocardiogr 1998;11:683-92.

45 Bach DS, Armstrong WF, Donovan CL, et al. Quantitative Doppler tissue imaging for assessment of regional myocardial velocities during transient ischaemia and reperfusion. Am Heart F 1996;132:721-5.

46 Von Bibra H, Tuchnitz A, Firschke C, et al. Doppler tissue imaging of left ventricular myocardium: initial results during pharmacologic stress [abstract]. I Am Coll Cardiol 1995;27(suppl):57A

47 Fontanet HL, Puelo JA, Davis MG, et al. Quantitative dobutamine stress echocardiography utilising Doppler tissue imaging [abstract]. F Am Coll Cardiol 1996;28(suppl):64A.

48 Payne N, Grocott-Mason R, Ionescu A, et al. Normal myocardial dose-response to dobutamine as assessed by tissue 1(suppl):S47.

49 Fraser A, Payne N, Grocott-Mason R, et al. Could stress echocardiography with off-line tissue Doppler quantificaechocardiography with off-line tissue Doppler quantificaEur f Echocardiogr 1999;1(suppl):S48.

50 Grocott-Mason R, Payne N, Wilkenshoff U, et al. Can off-line tissue Doppler echocardiography make dobutamine stress echocardiography objective? Eur Heart $\mathcal{f}$ 1999;20(suppl):687.

51 Pasquet A, Armstrong G, Beachler L, et al. Use of segmenal tissue Doppler velocity to quantitate exercise echocardiography. F Am Soc Echocardiogr 1999;12:901-12.

52 Hoffman JI. Transmural myocardial perfusion. Prog Cardiovasc Dis 1987;29:429.

53 Garcia MJ, Thomas JD, Klein AL. New doppler echocardiographic applications for the study of diastolic function. F Am Coll Cardiol 1998;32:865-75.

54 Farias CA, Rodriguez L, Garcia MJ, et al. Assessment of diastolic function by tissue doppler echocardiography: comparison with standard transmitral and pulmonary venous flow. F Am Soc Echocardiogr 1999;12:609-17.

55 Oki T, Tabata T, Yamada H, et al. Clinical application of pulsed Doppler tissue imaging for assessing abnormal left

56 Sohn DW, Chai IH, Lee DJ, et al. Assessment of mitral annulus velocity by doppler tissue imaging in the evaluation of left ventricular diastolic function. $\mathcal{F} \mathrm{Am}$ Coll Cardiol 1997;30:474-80.

57 Garcia MJ, Rodriguez L, Ares M, et al. Differentiation of constrictive pericarditis from restrictive cardiomyopathy: assessment of left ventricular diastolic velocities in longitudinal axis by Doppler tissue imaging. $\mathcal{F} \mathrm{Am}$ Coll Cardiol 1996;27:108-14.

58 Palka P, Lange A, Fleming AD, et al. Differences in myocardial velocity gradient measured throughout the cardiac cycle in patients with hypertrophic cardiomyopathy, athletes and patients with left ventricular hypertrophy due to hypertension. $\mathcal{F}$ Am Coll Cardiol 1997;30:760-8.

59 Sohn D-W, Song J-M, Zo J-H, et al. Mitral annulus velocity in the evaluation of left ventricular diastolic function in atrial fibrillation. F Am Soc Echocardiogr 1999;12:927-31.

60 Nishimura RA, Housmans PR, Hatle LK, et al. Assessment of diastolic function of the heart: background and current applications of Doppler echocardiography. Part 1. Physiologic and pathophysiologic features. Mayo Clin Proc 1989;64:71-81

61 Zamorano J. Is tissue Doppler echocardiography ready for clinical application? Eur Heart f 1999;20:558-60.

62 Mele D, Olstad B, Donateo M, et al. Strain rate imaging can accurately identify infarct segments in patients with myocardial infarction: a clinical study [abstract]. $7 \mathrm{Am} \mathrm{Coll}$ Cardiol 2000;35(suppl A):494A.

63 Pasquet A, Rimmerman C, Thomas J, et al. Is strain rate useful to quantitate exercise induced ischaemia: a SPECT validation study [abstract]. Eur f Echocardiogr 1999; 1(suppl):S48.

64 Cain P, Short L, Dart J, et al. Regional assessment by myocardial strain rate may offer a fully quantitative approach to dobutamine stress echo [abstract]. Eur $\mathcal{F}$ Echocardiogr 1999;1(suppl):S62.

65 Pasquet A, Flachskampf F, Odabashian J, et al. Can myocardial train recognise myocardial viability during low dose dobutamine stress echo? Eur $\mathcal{F}$ Echocardiogr 1999;1(suppl):S6. 